Chapter 12

\title{
Brain Computer Interface for Epilepsy Treatment
}

\author{
L. Huang and G. van Luijtelaar \\ Additional information is available at the end of the chapter \\ http://dx.doi.org/10.5772/55800
}

\section{Introduction}

A brain computer interface (BCI) is a communication system converting neural activities into signals that can control computer cursors or external devices (Fetz, 2007). BCI was initially and mainly employed for patients with severe motor disorders such as amyotrophic lateral sclerosis (ALS) by providing non-muscular bidirectional communication and control. However, the application of BCI has been extended to control various EEG signals for therapeutic purposes, such as seizure control in epilepsy patients. Although such BCIs did not demonstrate rapid control as in non-muscular communication, it still assumes that EEG based bidirectional control is possible (Wolpaw et al., 2002). More specifically, a BCI in epilepsy research, as in the current chapter, refers to a communication system capable to acquire signal and to implement real-time seizure detection/prediction and contingent delivery of warning stimuli or therapies such as electrical stimulation to control seizures (see the diagram). Such systems became feasible with technological development, and have been implemented in animal and human to control seizures. In the current chapter we will first give an overview of application of BCI, especially with deep brain stimulation in epilepsy research. Then we will discuss different components of a BCI system: input (signal acquisition), algorithm (seizure detection/prediction) and output (application and users), in particular stressing some important issues on BCI performance.

\section{BCI application in epilepsy research}

\subsection{Introduction of epilepsy treatment}

Epilepsy is a common chronic neurological disorder that afflicts $0.5-1 \%$ of the world's population (Hauser et al., 1993). More than one third patients do not respond to the antiepileptic 


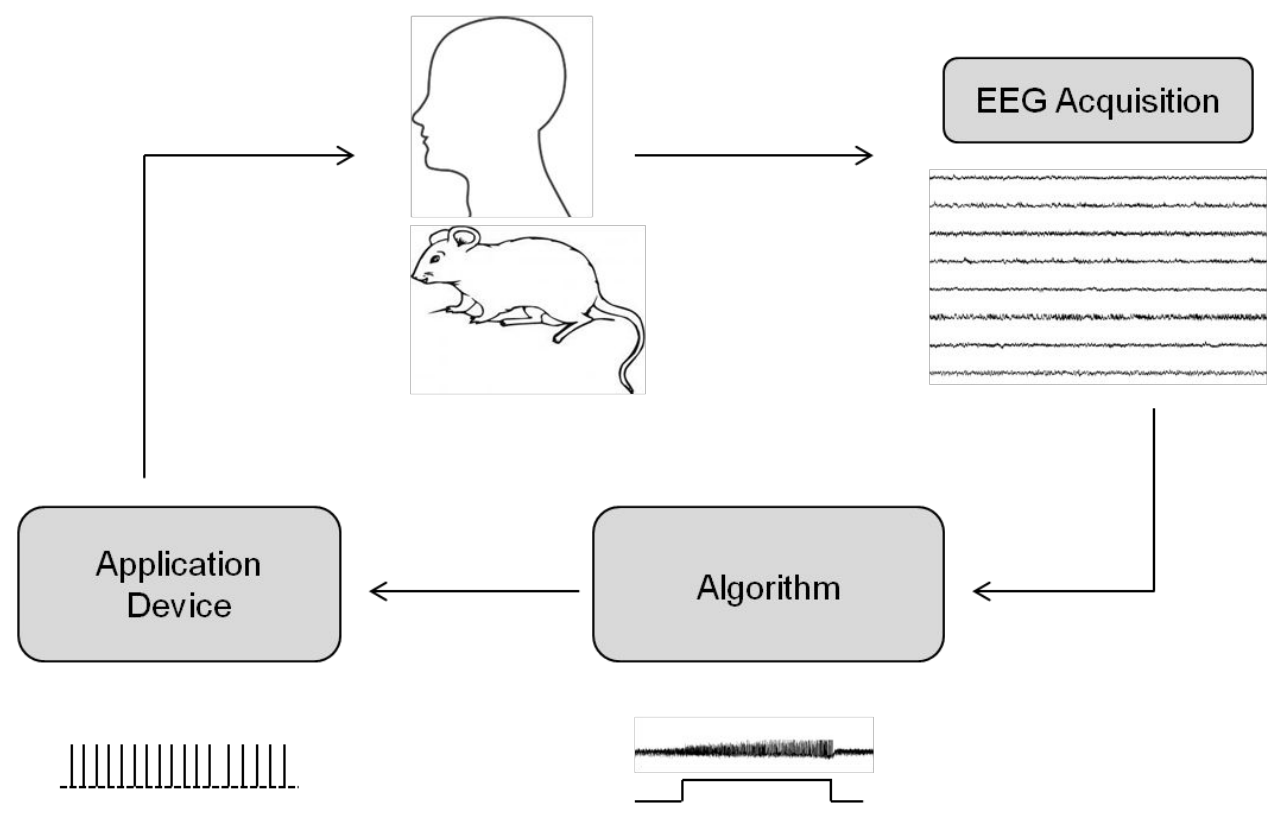

Diagram 1. A brain-computer interface $(\mathrm{BCl})$ in epilepsy research. $\mathrm{A} B \mathrm{BCl}$ system here has three components: input to acquire EEG signal (obtained by implanted electrodes or scalp electrodes, amplified, band-pass and notch filtered, and at the end was digitized), an algorithm to distinguish a seizure event from non-seizure state (seizure detection or prediction algorithm), and output of application device to deliver warning or therapies such as electrical stimulation to subjects in order to disrupt or modulate seizures.

drugs (AEDs) (Kwan and Brodie, 2000). Resective surgery, the main treatment for drug resistant patients, is not proper for certain patients such as those who have multiple foci or have generalized seizures without a clear local origin. In addition, it might be accompanied with some post-operation complications such as memory, language, sensory or motor deficits (Engel et al., 2003). Alternative treatments such as neurostimulation have been developed for the treatment of these patients. Deep brain stimulation (DBS), one type of neurostimulation, delivers electrical current to neural tissue to achieve therapeutic effects. Compared to surgery, DBS is less invasive, reversible and has the potential to customize treatment to individual patients. Following its success in the treatment of movement disorders (Nguyen et al., 2000; Pollak et al., 2002; Volkmann, 2004), DBS has received increasing attention as a viable treatment option for patients with refractory epilepsy.

While stimulation is conventionally delivered at the predefined protocol (scheduled stimulation), stimulation integrated in a BCI system is delivered depending on the neurophysiologic state of the brain (responsive stimulation). Such responsive stimulation as output application of BCI has the potential of advantages such as targeting seizure dynamics with higher temporal specificity, minimization of its side effects, reduction of neural tissue damage and of course a longer battery life. The following section will review research of responsive stimulation by BCI in animals and human respectively. 


\subsection{Responsive stimulation by BCI in animals}

Within the framework of a BCI concept, early neurophysiologic studies mainly adopted detection algorithm and feedback control to examine the effect of responsive stimulation on interictal spikes or seizure-like activities in slices. Psatta and colleagues (1983) delivered low frequency stimulation $(5 \mathrm{~Hz})$ to the caudate nucleus by feedback control in the epileptic foci in adult cats. Durand and his group (Kayyali and Durand, 1991; Nakagawa and Durand, 1991; Warren and Durand, 1998) applied current with feedback control in the focal area in hippocampal slices of rats. Meanwhile, Gluckman and colleagues (2001) applied a direct current (DC) electric field to hippocampal slices by using a computer controlled feedback algorithm. The outcomes of all these studies suggested antiepileptic effects of responsive stimulation by BCI.

Further, BCI with stimulation output was tested in vivo. Stimulation was applied to the motor cortex, the focus in the penicillin induced seizure model in rats by using proportional feedback control (Colpan et al., 2007). The results showed a significant reduction of mean amplitudes of seizures, suggesting positive effects of stimulation. Studies in a genetic absence epilepsy model (GAERS rats) were aimed at the search for optimal stimulation parameters of stimulation of the substantia nigra reticularis. Bilateral, bipolar and monophasic stimulation at $60 \mathrm{~Hz}$ and with $60-\mu$ s pulse width was optimal in the interruption of typical spike-wave discharges for absence epilepsy. However, when used for repeated stimulations, long-term suppression did not occur and even the number of spike-wave discharges increased (Feddersen et al., 2007). Stimulation by BCI was also tested with an automated detection program in the same model (Nelson et al., 2011a). Three types of stimulation frequency were investigated $(130,500$, $1000 \mathrm{~Hz}$ ) and the two high frequencies were more effective to reduce the duration of spikewave discharges.

BCI with a prediction algorithm was also applied in experimental epilepsy animal models. Stimulation was delivered in the hippocampus during the preictal period in the status epilepticus (SE) model in rats (Nair et al., 2006). The preliminary results showed a reduction in seizure frequency and longer seizure free periods, indicating antiepileptic effects of responsive hippocampal stimulation. Recently, BCI with a seizure prediction algorithm was also implemented in the SE model of TLE in six rats by delivering stimulation in the centromedial thalamus (Good et al., 2009). Apart from responsive stimulation, the study also tested scheduled stimulation. BCI with a seizure prediction algorithm was implemented in a penicillin induced seizure model in rats with low frequency stimulation $(1 \mathrm{~Hz})$ in the cortex (Wang et al., 2012), comparing with the scheduled stimulation and non-stimulation group. The outcomes of the last two studies favored responsive stimulation over scheduled stimulation, supporting BCI with stimulation output in seizure control.

Apart from conventional deep brain stimulation, application of BCI was recently extended to low frequency transcranial electrical stimulation (TES) in another rat model of generalized absence epilepsy (Berenyi et al., 2012). The results proved positive outcomes of responsive TES by $\mathrm{BCI}$ in terms of reducing the duration of spike-wave discharges without a subsequent rebound. 


\subsection{Responsive stimulation by BCI in human}

In clinic, some early patient studies have used afterdischarges (ADs) - elicited during routinely functional mapping - as model of seizures to investigate the effects of responsive stimulation as ADs are epileptiformic activities and can evolve into seizures. The promising results from some early studies (Lesser et al., 1999; Motamedi et al., 2002) justify the application of BCI in patients.

Osorio and his group (1998) developed a generic seizure detection algorithm by using wavelet analysis for early detection of seizure events. Based on that, Peters and colleagues (2001) described a BCI system- the first bedside prototype - integrated with such a real-time seizure detection algorithm and contingent delivery of stimulation at or near seizure onset. The performance and safety of this BCI system was further evaluated in eight patients (Osorio et al., 2005). The study demonstrated that BCI with stimulation output near seizure onset was practicable in real time and could be applied in a reliable and safe manner.

Following the success of BCI with stimulation application in seizure control, there is recognition of the need of implantable circuitry for clinical use. Recently, the first implantable BCI system - responsive neurostimulator (RNS) system (Fig 1) has been developed (NeuroPace, Mountain View, CA) in order to automatically detect early seizure events and deliver contingent stimulation. As proof of principle, Kossof and his group (2004) first evaluated the safety of BCI with output of an external stimulator in four epilepsy patients. While two patients had brief transient side effects, stimulation in general was well tolerated and safe. Although the efficacy of stimulation was not the aim of that study, electrographic seizures were altered and suppressed in these patients. Consistent with this study, Fountas and colleagues (2005) using an implantable BCI device, delivered responsive stimulation in eight patients. Seven out of eight patients displayed more than a $45 \%$ decrease in seizure frequency. Using the same BCI system, Smith and colleagues (2010) delivered responsive insular stimulation in one patient with refractory focal epilepsy after the resection of focal insular area. The patient showed a $50 \%$ reduction in seizure frequency after responsive stimulation. In another case report (Enatsu et al., 2012), a TLE patient received responsive stimulation (200 Hz) with RNS system in bilateral mesial temporal areas. An up to $50 \%$ decrease in seizure frequency was reported after delivery of stimulation.

Morrell and colleagues (2011) evaluated the efficacy and safety of the RNS device in a multicenter, double-blind, randomized controlled trial in 191 patients. These patients had improved quality of life and tolerated the treatment without obvious mood or cognitive adverse effects. In addition, seizures were significantly reduced for a 12-week blind period in the treatment group. Thus, the implantable BCI can be a promising therapeutic avenue for epilepsy treatment.

\section{The components of a BCI}

Like any other BCI systems, the BCI system discussed here consists of an input to obtain signal, algorithm to detect or predict seizures and output to apply warning or therapies to users. 


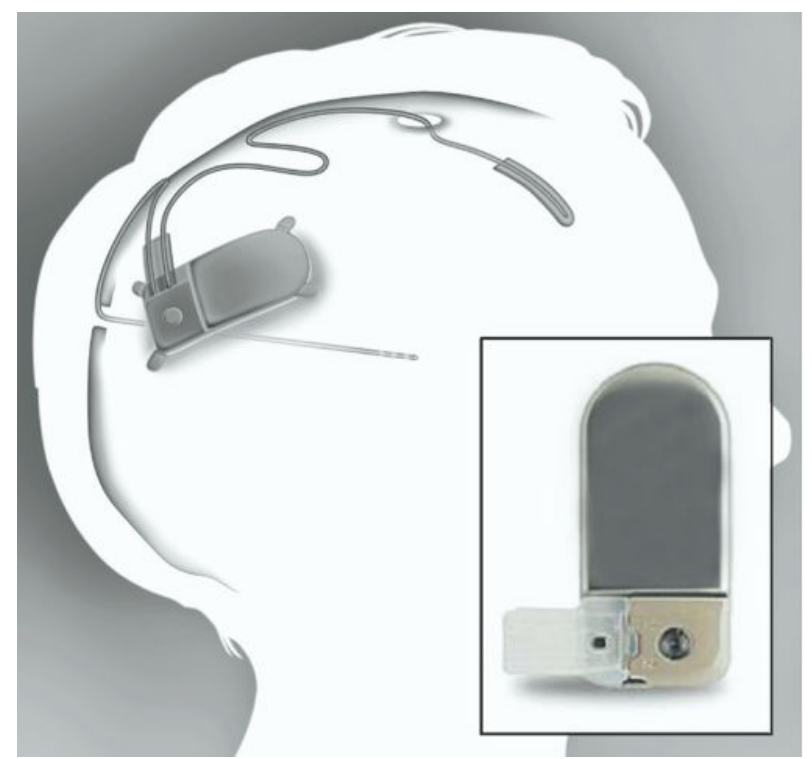

Figure 1. schematic graph of an implanted RNS stimulator, depth lead and cortical strip lead (NeuroPace, Mountain View, CA). The RNS neurostimulator (inset) has up to two leads. Either depth lead or cortical (subdural) strip lead is used in the system. Each has four electrode contacts that can be used for sensing and delivering stimulation. To obtain early seizure detection and delivery of focal electrical stimulation, leads are placed close to the seizure focus (Morrell et al, 2011).

\subsection{Signal acquisition}

The purpose of input in the BCI system discussed here is to acquire signal relevant for seizure detection and subsequent delivery of warning and therapy. The input is normally EEG recorded from the scalp or within the brain. The chosen input is acquired by electrode, then amplified and digitized in the BCI system. Recently, extracerebral signals such as cardiac (heart rate) or motor signals (speed, direction and joint movement) have emerged as a promising direction for seizure detection (Osorio and Schachter, 2011). Signals can be categorized as noninvasive (scalp EEG, extracerebral) or invasive (intracranial EEG). Scalp electrodes are the common sources of signals in clinic. In most cases, intracranial electrode allow better for early detection than scalp electrode, especially for partial seizures (Jouny et al., 2011).

One important factor that could affect $\mathrm{BCI}$ in signal acquisition operation is localization of ictal onset zone. Ictal onset zone is the area that generates the epileptic seizures. The ictal zone can be regional or broad, differing from one subject to another. A seizure event is more likely to be detected early after an ictal onset zone has been identified. One way to improve the localization of the ictal zone is to develop high-density scalp EEG recordings. These high density recordings provide higher spatial resolution with possibilities for dipole source localization compared to the more classical macroelectrode EEG recording systems. Intracranial recordings with microelectrode arrays on suspected candidate brain regions allow for a next improvement in localization. Information obtained with intracranial EEG recordings are 
considered the golden standard as far as spatial resolution of the source of epileptiformic activity is concerned. These intracranial recordings are also used for better understanding the interaction of local networks in the course of a seizure event (Jouny et al., 2011). With help of these new electrode recording systems, an ictal onset zone is much more likely to be identified correctly.

Another fundamental issue in BCI in this stage involves which signal to look at besides seizures, particularly for the purpose of the efficacy of therapy. For example, some interictal spikes are similar to seizures but last for only a few seconds without developing into a seizure (Jouny et al., 2011). Despite their short duration, they share similar characteristics components with a seizure. Highly localized activity such as microseizures might be correlated with partial seizure (Stead et al., 2010). If that holds true, these microseizures might be potential candidates for stimulation therapy.

\subsection{Seizure detection/prediction algorithm}

In the BCI system discussed here, algorithms should be able to detect a seizure event from non-seizure state (detection algorithm) or predict an upcoming seizure event (prediction algorithm). Quite some detection algorithms using various linear and nonlinear methods have been reported and obtained with various degrees of success. The validity and reliability of prediction algorithms remain questionable, despite some promising results (Mormann et al., 2007) as most of them were based on selective or limited EEG recordings or without statistical validation on sensitivity and specificity of each method (Carney et al., 2011).

Early detection is one issue that is less challenging but currently still far from being optimal for application in BCI research; it determines the time window in which warning or therapeutic devices are triggered. For warning purpose, the ideal condition is to detect seizure event before subjects lose awareness or consciousness. For therapy purpose, it requires to detect seizure as early as possible. As mentioned earlier, the use of high density arrays or microelectrodes are involved in signal acquisition and detection process; they favorably influence the ability to detect seizures early. The capability of an algorithm per se can directly determine how early a seizure event is detected. On the other hand, early detection always comes at the cost of specificity of an algorithm. Some improvements can be made to enhance the quality of early detection, such as using combination of linear and nonlinear features, multi-channel approach and decision making procedure (Jouny et al., 2011).

Another difficulty for BCI in algorithm operation is to include interaction of different states such as circadian fluctuations or vigilance state and seizures. Seizure probability can be affected by sleep stage, sleep debt and diurnal and other physiological rhythms (Haut et al., 2007; Malow, 2005). Circadian phases (Quigg et al., 2000; Smyk et al., 2012; Van Luijtelaar and Coenen, 1988) and sleep stage (Shouse et al., 2004; van Luijtelaar and Bikbaev, 2007) have been reported to have effects on seizures in rat models of epilepsy. Moreover, false positives by prediction algorithms were also found to favor some states of vigilance, in particular deep sleep (Navarro et al., 2005; Schelter et al., 2006). More efforts such as computational modeling need to be taken to probe states of vigilance and circadian phase to improve prediction of seizures. 


\subsection{Application and users}

The output of $\mathrm{BCI}$ here is the device that can trigger warning or therapies to users. Given its therapeutic purpose, therapy efficacy is the standard tool to evaluate BCI performance. In addition to electrical stimulation, other therapies have also been used such as anti-seizure compounds (Stein et al., 2000), thermal energy (cooling) (Hill et al., 2000), and operant conditioning (Osterhagen et al., 2010; Sterman, 2000). Compared to current, the main limitation of pharmacological and thermal energy therapy is their slow tissue diffusivity, which means that therapies will be delivered or arrive late to fully control the target area, even when delivered locally (Osorio and Frei, 2009). On the other side, electrical stimulation is the most common application of $\mathrm{BCI}$ and is also our focus in this section.

\subsubsection{Therapy parameters for users}

One challenge for $\mathrm{BCI}$ in application is how to stimulate, that is, selection of proper stimulation parameters. Stimulation parameters in human are often determined by trial and error with adjustment to avoid side effects while respecting safety limits for charge density (Sun et al., 2008). A number of studies especially in animals explored which stimulation parameters stimulation frequency, waveform, amplitude, duration- can result in better seizure control.

Stimulation frequency seems to be a key factor. High frequency stimulation (HFS) (> $100 \mathrm{~Hz})$ is typically used in clinic for treatment of movement disorders such as Parkinson's disease (Pollak et al., 2002; Volkmann, 2004) and also used for treatment of patients with refractory epilepsy. HFS has been delivered in various brain areas such as the anterior nucleus of the thalamus, hippocampus and thalamus (Andrade et al., 2006; Fisher et al., 2010; Velasco et al., 2000a; Velasco et al., 2000b) and has achieved various degree of success in seizure control.

In contrast, low frequency stimulation has limited and mixed effects. Stimulation at low frequency $(0.5-3 \mathrm{~Hz})$ in kindled animal models were found to raise the threshold of ADs (Carrington et al., 2007; Gaito et al., 1980; Ghorbani et al., 2007; Goodman et al., 2005). Yamamoto and colleagues (2002) demonstrated that LFS in the cortex had antiepileptic effects in the focus in patients with TLE. However, no other studies have further reported such effects of LFS in animal models and human of TLE. Even some clinical study reported contradictory evidence - aggravation of seizures by LFS on the centromedian thalamic nucleus in 12 patients (Velasco et al., 1997).

Furthermore, a few studies compared HFS and LFS under the same experimental condition. Albensi and colleagues (2004) compared the effects of HFS $(100 \mathrm{~Hz})$ and LFS $(1 \mathrm{~Hz})$ on epileptiform activities in hippocampal slices. Both types of stimulation suppressed epileptiform activities but with one difference: the onset of suppression by LFS was gradual but persistent while that of suppression by HFS was rapid but transient. The effects of LFS (5 Hz) and HFS (130 $\mathrm{Hz}$ ) were compared on ADs in kindled rats (Wyckhuys et al., 2010b). HFS was more effective with higher ADs threshold and longer latency. Boex and colleagues (2007) compared both types of stimulation on three patients and found that HFS was more effective in reducing seizure rate. Rajdev and colleagues (2011) examined stimulation frequency (high, medium and low), pulse width (high and low) and amplitude (high and low) in seizures in kainite treated rats. The results 
showed that low $(5 \mathrm{~Hz})$ and high frequency $(130 \mathrm{~Hz})$ were effective to suppress epileptic activities compared to medium frequency $(60 \mathrm{~Hz})$. Taken together, these studies suggested that HFS is most effective in seizure suppression while LFS has rather complicated effects depending on which animal model and epilepsy type is chosen for LFS application.

Other factors such as pulse width and waveform could also affect the effects of stimulation. In the aforementioned study, Rajdev and colleagues (2011) investigated the influences of different pulse widths (60, 120, 240 us) on ADs. With increase of pulse width (120, 240 us), less stimulation amplitude was needed to evoke ADs, indicating higher threshold of AD interruptions for shorter pulse width (60 us). The power of the pulse (intensity times duration) determines its efficacy in this model. In addition, the waveform of stimulation can also act on the effects of stimulation. The threshold for suppression of both somatic and axonal activity was lower with sinusoidal HFS than pulse train HFS (Jensen and Durand, 2007).

Recently, a new method of stimulation has been proposed: 'temporal coding'. Cota and colleagues (2009) reported that stimulation with a 'pseudo-randomized inter stimulus interval' significantly increased the threshold to tonic-clonic seizures in the pentylenetetrazole (PTZ) model. Similarly, Poisson distributed stimulation was found to reduce spontaneous seizures in the SE model of TLE (Wyckhuys et al., 2010a) and in the GEARs model of absence epilepsy (Nelson et al., 2011a). Further, cortical stimulation at multiple stimulation sites was investigated in different combinations of periodic/aperiodic (periodic - with fixed inter stimulus interval) and synchronous/ asynchronous stimulation manner (synchronous - stimulation at the same time) in rats (Nelson et al., 2011b). The results showed that asynchronous stimulation was more effective in suppression of seizure severity and duration.

Although it remains unclear which parameters are the best for which seizure model, these studies give us more insights on how to improve efficacy of therapy.

\subsubsection{Selection of target area}

Another basic question regarding BCI in application is where to stimulate. So far a variety of areas have been investigated for the effects of stimulation. These areas are either areas where seizures are generated or areas which are involved in progression of seizures. The intrinsic and extrinsic characteristics of these areas determine their different responses to stimulation. In each target area, for example, the constitutions of various neuron types and their biophysical properties are different, affecting responses of individual neuron and neuronal ensemble to stimulation. Besides, the anatomical connections among these target areas vary from each other, which can influence the dynamics of neuronal populations and sensitivity to stimulation (Sunderam et al., 2010).

\subsubsection{Patient-specific therapy}

It is also true that patient-specific therapy is the new direction for $\mathrm{BCI}$ in application operation. In clinic, stimulation parameters are usually adjusted in individual patients to avoid adverse effects while maintaining therapeutic effects. Osorio and his group (Osorio et al., 2010) used linear regression to examine in a retrospective study the results of a trial in which responsive 
stimulation was applied in eight patients with refractory epilepsy. Regression models tested the contributions of multiple potentially relevant factors such as parameter configurations (that is, different combinations of stimulation frequency, current intensity, duration, pulse width and location) to changes of seizure severity. The results showed that certain parameter configurations were more effective in reduction of seizure severity in some patients but not in others, indicating individual difference in parameters setting for stimulation. Thus adaptive, patients-specific stimulation settings might be a necessary new step for BCI in application in order to achieve optimal efficacy.

\section{Conclusion}

Unlike conventional BCIs, a BCI system in epilepsy emphasizes EEG-based communication for therapeutic purposes. The need of $\mathrm{BCI}$ rises from delivery of contingent therapies such as stimulation to control seizures. Although a large body of work has been done to explore contingent stimulation with help of BCI for epilepsy research in animal and human, it is still in an experimental stage. With development of technique in engineering and computer science, $\mathrm{BCI}$ is at the stage of becoming feasible for application of therapy in the treatment of patients in some forms of refractory epilepsy.

Future progress depends on the following crucial factors: development of electrode arrays or microelectrode, improvement of early detection, algorithms embedding variables such as vigilant states and circadian phases, exploration of patient-specific optimization of parameters, selection of proper target areas to maximize BCI performance. The development of BCI is an interdisciplinary challenge requiring vigorous and continuous efforts on relevant fields such as neuroscience, computer science, mathematics, and engineering. Although BCI in epilepsy research is still developing, it provides a promising therapeutic option to those who do not respond to conventional treatment.

\section{Author details}

L. Huang and G. van Luijtelaar

Dept Biological Psychology, Donders Center for Cognition, Donders Institute for Brain Cognition and Behaviour, Radboud University Nijmegen, Nijmegen, the Netherlands

\section{References}

[1] Albensi, B. C, Ata, G, Schmidt, E, Waterman, J. D, \& Janigro, D. (2004). Activation of long-term synaptic plasticity causes suppression of epileptiform activity in rat hippocampal slices. Brain Res. , 998, 56-64. 
[2] Andrade, D. M, Zumsteg, D, Hamani, C, Hodaie, M, Sarkissian, S, Lozano, A. M, \& Wennberg, R. A. (2006). Long-term follow-up of patients with thalamic deep brain stimulation for epilepsy. Neurology. , 66, 1571-3.

[3] Berenyi, A, Belluscio, M, Mao, D, \& Buzsaki, G. (2012). Closed-loop control of epilepsy by transcranial electrical stimulation. Science. , 337, 735-7.

[4] Boex, C, Vulliemoz, S, Spinelli, L, Pollo, C, \& Seeck, M. (2007). High and low frequency electrical stimulation in non-lesional temporal lobe epilepsy. Seizure. , 16, 664-9.

[5] Carney, P. R, Myers, S, \& Geyer, J. D. (2011). Seizure prediction: methods. Epilepsy Behav. 22 Suppl 1, S, 94-101.

[6] Carrington, C. A, Gilby, K. L, \& Mcintyre, D. C. (2007). Effect of focal low-frequency stimulation on amygdala-kindled afterdischarge thresholds and seizure profiles in fast- and slow-kindling rat strains. Epilepsia. , 48, 1604-13.

[7] Colpan, M. E, Li, Y, Dwyer, J, \& Mogul, D. J. (2007). Proportional feedback stimulation for seizure control in rats. Epilepsia. , 48, 1594-603.

[8] Cota, V. R. Medeiros Dde, C., Vilela, M.R., Doretto, M.C., Moraes, M.F., (2009). Distinct patterns of electrical stimulation of the basolateral amygdala influence pentylenetetrazole seizure outcome. Epilepsy Behav. 14 Suppl , 1, 26-31.

[9] Enatsu, R, Alexopoulos, A, Bingaman, W, \& Nair, D. (2012). Complementary effect of surgical resection and responsive brain stimulation in the treatment of bitemporal lobe epilepsy: a case report. Epilepsy Behav. , 24, 513-6.

[10] Engel, J. Jr., Wiebe, S., French, J., Sperling, M., Williamson, P., Spencer, D., Gumnit, R., Zahn, C., Westbrook, E., Enos, B., (2003). Practice parameter: temporal lobe and localized neocortical resections for epilepsy. Epilepsia. , 44, 741-51.

[11] Feddersen, B, Vercueil, L, Noachtar, S, David, O, Depaulis, A, \& Deransart, C. (2007). Controlling seizures is not controlling epilepsy: a parametric study of deep brain stimulation for epilepsy. Neurobiol Dis. , 27, 292-300.

[12] Fetz, E. E. (2007). Volitional control of neural activity: implications for braincomputer interfaces. J Physiol. , 579, 571-9.

[13] Fisher, R, Salanova, V, Witt, T, Worth, R, Henry, T, Gross, R, Oommen, K, Osorio, I, Nazzaro, J, Labar, D, Kaplitt, M, Sperling, M, Sandok, E, Neal, J, Handforth, A, Stern, J, Desalles, A, Chung, S, Shetter, A, Bergen, D, Bakay, R, Henderson, J, French, J, Baltuch, G, Rosenfeld, W, Youkilis, A, Marks, W, Garcia, P, Barbaro, N, Fountain, N, Bazil, C, Goodman, R, \& Mckhann, G. Babu Krishnamurthy, K., Papavassiliou, S., Epstein, C., Pollard, J., Tonder, L., Grebin, J., Coffey, R., Graves, N., (2010). Electrical stimulation of the anterior nucleus of thalamus for treatment of refractory epilepsy. Epilepsia. , 51, 899-908. 
[14] Fountas, K. N, Smith, J. R, Murro, A. M, Politsky, J, Park, Y. D, \& Jenkins, P. D. (2005). Implantation of a closed-loop stimulation in the management of medically refractory focal epilepsy: a technical note. Stereotact Funct Neurosurg. , 83, 153-8.

[15] Gaito, J, Nobrega, J. N, \& Gaito, S. T. (1980). Interference effect of 3 Hz brain stimulation on kindling behavior induced by $60 \mathrm{~Hz}$ stimulation. Epilepsia. , 21, 73-84.

[16] Ghorbani, P, Mohammad-zadeh, M, Mirnajafi-zadeh, J, \& Fathollahi, Y. (2007). Effect of different patterns of low-frequency stimulation on piriform cortex kindled seizures. Neurosci Lett. , 425, 162-6.

[17] Gluckman, B. J, Nguyen, H, Weinstein, S. L, \& Schiff, S. J. (2001). Adaptive electric field control of epileptic seizures. J Neurosci. , 21, 590-600.

[18] Good, L. B, Sabesan, S, Marsh, S. T, Tsakalis, K, Treiman, D, \& Iasemidis, L. (2009). Control of synchronization of brain dynamics leads to control of epileptic seizures in rodents. Int J Neural Syst. , 19, 173-96.

[19] Goodman, J. H, Berger, R. E, \& Tcheng, T. K. (2005). Preemptive low-frequency stimulation decreases the incidence of amygdala-kindled seizures. Epilepsia. , 46, 1-7.

[20] Hauser, W. A, Annegers, J. F, \& Kurland, L. T. (1993). Incidence of epilepsy and unprovoked seizures in Rochester, Minnesota: 1935-1984. Epilepsia. , 34, 453-68.

[21] Haut, S. R, Hall, C. B, Masur, J, \& Lipton, R. B. (2007). Seizure occurrence: precipitants and prediction. Neurology. , 69, 1905-10.

[22] Hill, M. W, Wong, M, Amarakone, A, \& Rothman, S. M. (2000). Rapid cooling aborts seizure-like activity in rodent hippocampal-entorhinal slices. Epilepsia. , 41, 1241-8.

[23] Jensen, A. L, \& Durand, D. M. (2007). Suppression of axonal conduction by sinusoidal stimulation in rat hippocampus in vitro. J Neural Eng. , 4, 1-16.

[24] Jouny, C. C, Franaszczuk, P. J, \& Bergey, G. K. (2011). Improving early seizure detection. Epilepsy Behav. 22 Suppl 1, S, 44-8.

[25] Kayyali, H, \& Durand, D. (1991). Effects of applied currents on epileptiform bursts in vitro. Exp Neurol. , 113, 249-54.

[26] Kossoff, E. H, Ritzl, E. K, Politsky, J. M, Murro, A. M, Smith, J. R, Duckrow, R. B, Spencer, D. D, \& Bergey, G. K. (2004). Effect of an external responsive neurostimulator on seizures and electrographic discharges during subdural electrode monitoring. Epilepsia. , 45, 1560-7.

[27] Kwan, P, \& Brodie, M. J. (2000). Early identification of refractory epilepsy. N Engl J Med. , 342, 314-9.

[28] Lesser, R. P, Kim, S. H, Beyderman, L, Miglioretti, D. L, Webber, W. R, Bare, M, Cysyk, B, Krauss, G, \& Gordon, B. (1999). Brief bursts of pulse stimulation terminate afterdischarges caused by cortical stimulation. Neurology. , 53, 2073-81.

[29] Malow, B. A. (2005). Sleep and epilepsy. Neurol Clin. , 23, 1127-47. 
[30] Mormann, F, Andrzejak, R. G, Elger, C. E, \& Lehnertz, K. (2007). Seizure prediction: the long and winding road. Brain. , 130, 314-33.

[31] Morrell, M. J. (2011). Responsive cortical stimulation for the treatment of medically intractable partial epilepsy. Neurology. , 77, 1295-1304.

[32] Motamedi, G. K, Lesser, R. P, Miglioretti, D. L, Mizuno-matsumoto, Y, Gordon, B, Webber, W. R, Jackson, D. C, Sepkuty, J. P, \& Crone, N. E. (2002). Optimizing parameters for terminating cortical afterdischarges with pulse stimulation. Epilepsia. , $43,836-46$.

[33] Nair, S. P, Sackellares, J. C, Shiau, D. S, Norman, W. M, Dance, L. K, Pardalos, P. M, Principe, J. C, \& Carney, P. R. (2006). Effects of acute hippocampal stimulation on EEG dynamics. Conf Proc IEEE Eng Med Biol Soc. , 1, 4382-6.

[34] Nakagawa, M, \& Durand, D. (1991). Suppression of spontaneous epileptiform activity with applied currents. Brain Res. , 567, 241-7.

[35] Navarro, V, \& Martinerie, J. Le Van Quyen, M., Baulac, M., Dubeau, F., Gotman, J., (2005). Seizure anticipation: do mathematical measures correlate with video-EEG evaluation? Epilepsia. , 46, 385-96.

[36] Nelson, T. S, Suhr, C. L, Freestone, D. R, Lai, A, Halliday, A. J, Mclean, K. J, Burkitt, A. $\mathrm{N}$, \& Cook, M. J. seizure control with very high frequency electrical stimulation at seizure onset in the GAERS model of absence epilepsy. Int J Neural Syst. , 21, 163-73.

[37] Nelson, T. S, Suhr, C. L, Lai, A, Halliday, A. J, Freestone, D. R, Mclean, K. J, Burkitt, A. N, \& Cook, M. J. (2011b). Exploring the tolerability of spatiotemporally complex electrical stimulation paradigms. Epilepsy Res. , 96, 267-75.

[38] Nguyen, J. P, \& Lefaucher, J. P. Le Guerinel, C., Eizenbaum, J.F., Nakano, N., Carpentier, A., Brugieres, P., Pollin, B., Rostaing, S., Keravel, Y., (2000). Motor cortex stimulation in the treatment of central and neuropathic pain. Arch Med Res. , 31, 263-5.

[39] Osorio, I, Frei, M. G, \& Wilkinson, S. B. (1998). Real-time automated detection and quantitative analysis of seizures and short-term prediction of clinical onset. Epilepsia. , 39, 615-27.

[40] Osorio, I, Frei, M. G, Sunderam, S, Giftakis, J, Bhavaraju, N. C, Schaffner, S. F, \& Wilkinson, S. B. (2005). Automated seizure abatement in humans using electrical stimulation. Ann Neurol. , 57, 258-68.

[41] Osorio, I, \& Frei, M. G. (2009). Real-time detection, quantification, warning, and control of epileptic seizures: the foundations for a scientific epileptology. Epilepsy Behav. , 16, 391-6.

[42] Osorio, I, Manly, B, \& Sunderam, S. (2010). Toward a quantitative multivariate analysis of the efficacy of antiseizure therapies. Epilepsy Behav. , 18, 335-43.

[43] Osorio, I, \& Schachter, S. (2011). Extracerebral detection of seizures: a new era in epileptology? Epilepsy Behav. 22 Suppl 1, S, 82-7. 
[44] Osterhagen, L, Breteler, M, \& Van Luijtelaar, G. (2010). Does arousal interfere with operant conditioning of spike-wave discharges in genetic epileptic rats? Epilepsy Res. , 90, 75-82.

[45] Peters, T. E, Bhavaraju, N. C, Frei, M. G, \& Osorio, I. (2001). Network system for automated seizure detection and contingent delivery of therapy. J Clin Neurophysiol. , $18,545-9$.

[46] Pollak, P, Fraix, V, Krack, P, Moro, E, Mendes, A, Chabardes, S, Koudsie, A, \& Benabid, A. L. (2002). Treatment results: Parkinson's disease. Mov Disord. 17 Suppl 3, S, 75-83.

[47] Psatta, D. M. (1983). Control of chronic experimental focal epilepsy by feedback caudatum stimulations. Epilepsia. , 24, 444-54.

[48] Quigg, M, Clayburn, H, Straume, M, Menaker, M, \& Bertram, E. H. rd, (2000). Effects of circadian regulation and rest-activity state on spontaneous seizures in a rat model of limbic epilepsy. Epilepsia. , 41, 502-9.

[49] Rajdev, P, Ward, M, \& Irazoqui, P. (2011). Effect of stimulus parameters in the treatment of seizures by electrical stimulation in the kainate animal model. Int J Neural Syst. , 21, 151-62.

[50] Schelter, B, Winterhalder, M, Maiwald, T, Brandt, A, Schad, A, Timmer, J, \& Schulzebonhage, A. (2006). Do false predictions of seizures depend on the state of vigilance? A report from two seizure-prediction methods and proposed remedies. Epilepsia. , 47, 2058-70.

[51] Shouse, M. N, Scordato, J. C, \& Farber, P. R. (2004). Sleep and arousal mechanisms in experimental epilepsy: epileptic components of NREM and antiepileptic components of REM sleep. Ment Retard Dev Disabil Res Rev. , 10, 117-21.

[52] Smith, J. R, Fountas, K. N, Murro, A. M, Park, Y. D, Jenkins, P. D, Morrell, M, Esteller, R, \& Greene, D. (2010). Closed-loop stimulation in the control of focal epilepsy of insular origin. Stereotact Funct Neurosurg. , 88, 281-7.

[53] Smyk, M. K, Coenen, A, Lewandowski, M. H, \& Van Luijtelaar, G. (2012). Internal desynchronization facilitates seizures. Epilepsia. , 53, 1511-8.

[54] Stead, M, Bower, M, Brinkmann, B. H, Lee, K, Marsh, W. R, Meyer, F. B, Litt, B, Van Gompel, J, \& Worrell, G. A. (2010). Microseizures and the spatiotemporal scales of human partial epilepsy. Brain. , 133, 2789-97.

[55] Stein, A. G, Eder, H. G, Blum, D. E, Drachev, A, \& Fisher, R. S. (2000). An automated drug delivery system for focal epilepsy. Epilepsy Res. , 39, 103-14.

[56] Sterman, M. B. (2000). Basic concepts and clinical findings in the treatment of seizure disorders with EEG operant conditioning. Clin Electroencephalogr. , 31, 45-55.

[57] Sun, F. T, Morrell, M. J, \& Wharen, R. E. Jr., (2008). Responsive cortical stimulation for the treatment of epilepsy. Neurotherapeutics. , 5, 68-74. 
[58] Sunderam, S, Gluckman, B, Reato, D, \& Bikson, M. (2010). Toward rational design of electrical stimulation strategies for epilepsy control. Epilepsy Behav. , 17, 6-22.

[59] Van Luijtelaar, E. L, \& Coenen, A. M. (1988). Circadian rhythmicity in absence epilepsy in rats. Epilepsy Res. , 2, 331-6.

[60] Van Luijtelaar, G, \& Bikbaev, A. (2007). Midfrequency cortico-thalamic oscillations and the sleep cycle: genetic, time of day and age effects. Epilepsy Res. , 73, 259-65.

[61] Velasco, A. L, Velasco, M, Velasco, F, Menes, D, Gordon, F, Rocha, L, Briones, M, \& Marquez, I. and chronic electrical stimulation of the hippocampus on intractable temporal lobe seizures: preliminary report. Arch Med Res. , 31, 316-28.

[62] Velasco, M, Velasco, F, Velasco, A. L, Brito, F, Jimenez, F, Marquez, I, \& Rojas, B. (1997). Electrocortical and behavioral responses produced by acute electrical stimulation of the human centromedian thalamic nucleus. Electroencephalogr Clin Neurophysiol. , 102, 461-71.

[63] Velasco, M, Velasco, F, Velasco, A. L, Boleaga, B, Jimenez, F, Brito, F, \& Marquez, I. (2000b). Subacute electrical stimulation of the hippocampus blocks intractable temporal lobe seizures and paroxysmal EEG activities. Epilepsia. , 41, 158-69.

[64] Volkmann, J. (2004). Deep brain stimulation for the treatment of Parkinson's disease. J Clin Neurophysiol. , 21, 6-17.

[65] Wang, L, Guo, H, Yu, X, Wang, S, Xu, C, Fu, F, Jing, X, Zhang, H, \& Dong, X. (2012). Responsive electrical stimulation suppresses epileptic seizures in rats. PLoS One. 7, e38141.

[66] Warren, R. J, \& Durand, D. M. (1998). Effects of applied currents on spontaneous epileptiform activity induced by low calcium in the rat hippocampus. Brain Res. , 806, 186-95.

[67] Wolpaw, J. R, Birbaumer, N, Mcfarland, D. J, Pfurtscheller, G, \& Vaughan, T. M. (2002). Brain-computer interfaces for communication and control. Clin Neurophysiol. , 113, 767-91.

[68] Wyckhuys, T, Boon, P, Raedt, R, Van Nieuwenhuyse, B, Vonck, K, \& Wadman, W. (2010a). Suppression of hippocampal epileptic seizures in the kainate rat by Poisson distributed stimulation. Epilepsia. , 51, 2297-304.

[69] Wyckhuys, T, Raedt, R, Vonck, K, Wadman, W, \& Boon, P. (2010b). Comparison of hippocampal Deep Brain Stimulation with high $(130 \mathrm{~Hz})$ and low frequency $(5 \mathrm{~Hz})$ on afterdischarges in kindled rats. Epilepsy Res. , 88, 239-46.

[70] Yamamoto, J, Ikeda, A, Satow, T, Takeshita, K, Takayama, M, Matsuhashi, M, Matsumoto, R, Ohara, S, Mikuni, N, Takahashi, J, Miyamoto, S, Taki, W, Hashimoto, N, Rothwell, J. C, \& Shibasaki, H. (2002). Low-frequency electric cortical stimulation has an inhibitory effect on epileptic focus in mesial temporal lobe epilepsy. Epilepsia., 43, 491-5. 